Using data from the Medical Birth Register, we identified $2,777,045$ babies born to $1,417,903$ mothers during the study period. Of those 9,334 were born to women with $\mathrm{CH}$. Maternal CH were recorded using ICD-8, ICD-9 and ICD-10. Outcome measures were pre-eclampsia (PE), emergency and elective caesarean sections (CS), spontaneous preterm birth (PTB $<37$ weeks' gestation), medically indicated PTB, stillbirth and small for gestational age. Multivariate logistic regression models were performed using Stata 16 and adjusting for several socio-demographic and perinatal confounders.

Results Compared to normotensive women, we found higher odds among hypertensive women of the following outcomes: PE adjusted odds ratio [aOR (95\% confidence intervals)]: [4.60 (4.31, 4.92)]; emergency CS [1.64 (1.53, 1.77)]; elective CS [1.63 (1.51, 1.76)]; medically indicated PTB [3.36 (3.11, 3.63)]; stillbirth [1.62 (1.26, 2.08)] and SGA [2.33 (2.13, 2.54)]. Moreover, Women of advanced maternal age were more likely to have emergency CS [aOR: 1.82 (1.61, 2.05)]; elective CS $[1.83(1.64,2.04)]$; medically indicated PTB [4.01 $(3.54,4.54)]$ and SGA $[2.61(2.24,3.04)]$ compared to younger normotensive women. However, the magnitude of these associations appears to decrease over time apart of the association with $\mathrm{PE}$ which appears to have remained almost constant over time.

Conclusion Women with chronic hypertension are at increased risk of adverse maternal, fetal and neonatal outcomes and the risk differs according to maternal age. However, the effect of antihypertensive treatment and severity of hypertension have not been taken into account in this study.

\section{P43 EXPERIENCE OF CHILD WELFARE SERVICES AND LONG-TERM ADULT MENTAL HEALTH OUTCOMES: A SCOPING REVIEW}

S McKenna*, A Maguire, I Onyeka, M Donnelly. Centre for Public Health, Queen's University, Belfast, UK

\subsection{6/jech-2020-SSMabstracts. 137}

Background Research consistently suggests that children in care have higher rates of mental ill-health compared to their peers in the community, however, studies exploring the longterm adult mental health outcomes have shown mixed results. The proliferation of research in this area is challenging and to date no comprehensive overview exists. This study synthesised the literature on the mental health outcomes of adults with a history of child welfare involvement.

Methods A systematic scoping review methodology was used to search five electronic databases (MEDLINE, EMBASE, PsychINFO, IBSS, Social Policy and Practice). Two reviewers screened all papers independently. Studies were included if they examined any child welfare exposure (including being known to social services and remaining at home or being removed from the home and placed in foster care or residential care) and adult mental health status.

Results In total 4,591 records were retrieved, of which 53 met the eligibility criteria. Four major themes of child welfare and adult mental health research were identified based broadly on service type: 35 studies examined out-of-home care (OHC) without specifying outcomes by placement type; eleven studies of OHC examined specific placement types; four studies examined both in-home care (IHC) and OHC; and three studies examined IHC services only. Overall, both OHC and IHC were associated with an increased risk of adult mental illhealth, suicide attempt and completed suicide. Potential moderating factors such as gender and care related experiences have been explored but produced conflicting results. Reason for becoming known to child welfare services and experience of abuse or neglect have not been widely explored, nor have outcomes in those who received child welfare services but remained in their own home. Mental health was defined and measured heterogeneously and detail on welfare services received was lacking.

Conclusion This is the first review to systematically map the available evidence on child welfare exposure and adult mental health. There is a need for detailed, longitudinal studies to better understand the aetiology of mental ill health amongst adults with a childhood history of welfare service exposure, with more standardised measures of mental ill-health and more detail from authors on specific care exposure.

\section{P44 DIFFERENT PATTERNS OF FAMILY ADVERSITY IN EARLY CHILDHOOD AND MIDDLE CHILDHOOD MENTAL HEALTH: WHEN DOES CHILDCARE HAVE A PROTECTIVE EFFECT? RESEARCH USING THE GROWING UP IN SCOTLAND STUDY}

A Parkes*. MRC/CSO Social and Public Health Sciences Unit, University of Glasgow, Glasgow, UK

\subsection{6/jech-2020-SSMabstracts. 138}

Background Various patterns of early childhood family adversity characterised by poverty and/or other stressors are major risk factors for middle childhood mental health. Efforts to reduce mental health inequalities would benefit from understanding whether childcare moderates effects of different adversity patterns. This study examined hypotheses regarding centre-based (eg nursery) and non-centre (eg grandparent, childminder) childcare in: (1) buffering effects of adversity mainly characterised by poverty; (2) potentiating effects of adversity characterised by multiple stressors.

Methods We used the Growing $U p$ in Scotland first birth cohort (born in 2004/5, $\mathrm{n}=5217$ ), selecting families with a singleton birth, where mothers provided information on adversity and childcare at child age 10, 22 and 34 months, and where there was parent-reported information on children's externalizing and internalizing problems at 46, 58, 70, 94, 122 and/or 152 months $(n=3419)$. Using Mplus, latent class analysis of 19 indicators identified four adversity subtypes: Low (66\%), Health-related (20\%), Poverty/discord (9\%), Multiple $(5 \%)$. Growth mixture modelling identified five childcare patterns: Low (28\%), Moderate Non-Centre (30\%), High Non-Centre (16\%), High Centre (12\%), High Combined $(13 \%)$. Latent growth models of problem trajectories (approximate ages 4 to 12 years) on adversity, childcare and interaction terms controlled for child gender, low birth weight, mothers' age, ethnicity, education, smoking in pregnancy, family type and number of children at baseline.

Results Compared to the Low adversity subtype, children from higher-risk subtypes had higher 8.25-year intercepts of externalizing problems (coefficients with $95 \%$ confidence intervals: Health-related 1.46, 1.15-1.78; Poverty/discord 1.34, 0.82 1.87; Multiple 2.56, 1.86-3.26) and internalizing problems (Health-related 1.20, 0.93-1.47; Poverty/discord 1.16, 0.701.61; Multiple 2.60, 2.02-3.17); and steeper linear growth in 
internalizing problems (Health-related 0.08, 0.04-0.12; Poverty/discord 0.08, 0.01-0.16; Multiple 0.24, 0.15-0.34). Adding childcare and interactions with adversity yielded negative interaction terms for High Centre $\mathrm{x}$ Poverty/discord on the externalizing intercept $(-1.83,-3.18$ to -0.47$)$; and for High Combined $\mathrm{x}$ Poverty/discord on the externalizing intercept ($1.70,-3.23$ to -0.15$)$, internalizing intercept $(-1.52,-2.44$ to $0.60)$, and internalizing linear slope $(-0.23,-0.44$ to -0.02$)$. Corresponding interaction terms for Health-related adversity were generally small and negative, but for Multiple adversity were larger and all positive: for both these adversity subtypes, confidence intervals spanned zero. Sensitivity analyses incorporating teacher- and child-reported 122/152-month outcomes confirmed main findings.

Conclusion Centre-based care, especially when combined with individual non-parental care, buffered children's mental health from family adversity characterised by poverty and interparental discord. Limitations include low statistical power for small subgroups and lack of childcare quality information. Findings indicate childcare may be protective against some, but not all, patterns of family adversity.

\section{P45 EXERCISING TO CONTROL SIGNS AND SYMPTOMS OF STRESS AND DEPRESSION}

\begin{abstract}
1,2JA Martin*, 1,2,3 CE Gheorge, ${ }^{4} \mathrm{M}$ Molloy, ${ }^{5} \mathrm{~K}$ O'Halloran, ${ }^{1,2} \mathrm{TG}$ Dinan, ${ }^{1,2,3} \mathrm{JF}$ Cryan, $1,2,6 \mathrm{G}$ Clarke. 'Department of Psychiatry and Neurobehavioural Science, University College Cork, Cork, Ireland; ${ }^{2}$ APC Microbiome Ireland, University College Cork, Cork, Ireland; ${ }^{3}$ Department of Anatomy and Neuroscience, University College Cork, Cork, Ireland; ${ }^{4}$ Clinical Research Ethics Committee of the Cork Teaching Hospitals, University College Cork, Cork, Ireland; ${ }^{5}$ Department of Physiology, University College Cork, Cork, Ireland; ${ }^{6}$ INFANT Research Centre, University College Cork, Cork, Ireland
\end{abstract}

\subsection{6/jech-2020-SSMabstracts. 139}

Background 300 million people worldwide suffer from depression. A poorly understood stress-related pathophysiology is compounded by frequent treatment-resistance.

Undertaking regular exercise has consistently shown to prevent, protect and increase resilience against the development of aberrant stress-related responses seen in depression, whereas sedentary behaviour tends to heighten symptoms. Despite the promise shown by exercise its clinical use is undermined by poor understanding of how beneficial effects are produced.

Stress exposure can dysregulate important physiological pathways such as the hypothalamic-pituitary-adrenal (HPA) axis and the sympathetic nervous system. Disruption of tryptophan metabolism, including increased metabolism along the kynurenine pathway, is a neurobiological hallmark of depressed patients. Recent studies have shown that the beneficial impact of exercise for mood and cognition may be linked to reduced kynurenine production.

The aim was to examine the benefits of a 12 -week exercise programme in healthy sedentary adults across mood, stress, anxiety and depression were mediated by the regulation of tryptophan metabolism in a dose dependent manner.

Methods 32 healthy sedentary adults participated (16 females). To establish a baseline profile each participant undertook a battery of psychometric assessments, cognitive assessments and a cycle ergometer-based incremental fitness assessment. Measures of oxygen uptake and capillary blood lactate levels were taken at the end of each stage during the fitness assessment.
After the baseline assessment, participants were randomised into one of four groups (high, moderate, low dose exercise, or the sedentary control group). Exercise was administered over 3 sessions/week based on individual fitness and group. Monthly fitness and psychometric assessments were performed throughout to monitor change across the 12-week exercise programme. Data analyses were performed using SPSS.

Results As expect, participants showed a positive increase in fitness and performance with an increase in maximal oxygen uptake $(F(1,28)=14.62, p=0.001, \eta p 2=0.343)$ after 12 -weeks exercise training, accompanied by an increased relative peak power output $(\mathrm{rPPO})(\mathrm{F}(1,28)=16.93, \mathrm{p}=0.000, \eta \mathrm{p} 2=0.377)$ and an increased time to exhaustion (T2Ex) $(\mathrm{F}(1,28)=31.07$, $\mathrm{p}=0.000, \eta \mathrm{p} 2=0.526)$. Post hoc paired T-tests revealed that the most pronounced effects on rPPO and T2Ex occurred in the moderate or high intensity groups There was a reduction in scores on the Beck Depression Inventory (BDI) $(\mathrm{F}(1,28)$ $=6.50, \mathrm{p}=0.017, \eta \mathrm{p} 2=0.189)$ and the Perceived Stress Scale (PSS) $(\mathrm{F}(1,28)=4.09, \mathrm{p}=0.053, \eta \mathrm{p} 2=0.127)$ scores after 12 weeks of exercise.

Conclusion In summary, these results confirm that exercise training beneficially influences measures of stress and depression. Further work is required to understand the role of exercise-induced alterations in tryptophan metabolism in mediating these effects.

\section{P46 THE MEANING OF RECOVERY AT THE INTERSECTION OF ETHNICITY AND GENDER FOR AFRICAN AND CARIBBEAN MEN IN ENGLAND}

${ }^{1} \mathrm{~K}$ Southby*, ${ }^{2} \mathrm{~F}$ Keating, ${ }^{2} \mathrm{~S}$ Joseph. ${ }^{1}$ Centre for Health Promotion Research, Leeds Beckett University, Leeds, UK; ${ }^{2}$ Department for Social Work, Royal Holloway, University of London, London, UK

10.1136/jech-2020-SSMabstracts. 140

Background 'Recovery' is a contested concept within mental health. This is to the detriment of patients' progression. The aim of this paper is to consider the meaning of recovery for African and Caribbean men with mental health experience in England.

Methods A qualitative design using a phenomenological approach captured the dynamics of recovery processes and outcomes for African and Caribbean men across the two study sites (Leeds and London). Fifty-nine in-depth semi-structured interviews were conducted with African and Caribbean men with mental health experience $(n=30)$, supporters/family carers $(n=15)$, and service providers $(n=14)$. Data were analysed using Interpretative Phenomenological Analysis (IPA). At two co-creation events, service users co-analysed the data.

Results Findings suggest that recovery for African and Caribbean men is a complex, dynamic concept. A number of interconnected and often overlapping aspects collectively represent and contribute to recovery. These include recovery as a healing journey, leading a 'normal' life, autonomy and control, aspirations for the future, identity, and being free from health services.

Conclusion Whilst previous research similarly highlights issues of autonomy, social inclusion, personalisation, and identity as fundamental to recovery for all service users, this paper argues that recovery for African and Caribbean men is flavoured by their lived-experience at the intersections of ethnicity and gender. Service providers should acknowledge the men's personal and collective understanding of recovery based on their unique life histories. 